\title{
Análise de erros computacionais de um modelo generalizado da equação de Richards
}

\author{
Amauri A. de Freitas* ${ }^{*} \quad$ Daniel G. A. Vigo Marcello G.Teixeira \\ Depto. de Ciências de Computação - Instituto de Matemática, PPGI, UFRJ \\ Caixa Postal 68.530, CEP 21941-590, Cidade Universitária, Ilha do Fundão, Rio de Janeiro, RJ, Brasil \\ amauri.freitas@ppgi.ufrj.br, dgalfaro@dcc.ufrj.br, marcellogt@dcc.ufrj.br
}

Carlos A. B. de Vasconcellos

Depto. de Engenharia de Fortificação e Construção - Instituto Militar de Engenharia - IME

Praça General Tiburcio, 80, Praia Vermelha, Urca, CEP 22290-270, Rio de Janeiro, RJ, Brasil.

vasconcellos@ime.eb.br

\section{$\underline{\text { RESUMO }}$}

A infiltração de água no solo é um processo hidrológico cuja simulação computacional tem diversas aplicações em vários ramos da ciência, tais como irrigação, meio ambiente, petróleo e gás, geologia, agronomia dentre outras. A equação que governa esse fenômeno físico é a Equação de Richards, que pode ser escrita como

$$
\frac{\partial \theta}{\partial t}=\frac{\partial}{\partial x}\left[D(\theta) \frac{\partial \theta}{\partial x}\right]+\frac{\partial}{\partial z}\left[D(\theta) \frac{\partial \theta}{\partial z}\right]+\frac{\partial K(\theta)}{\partial z}
$$

onde $\mathrm{x}$ é a coordenada horizontal, $z$ é a coordenada vertical, $t$ é o tempo, $K(\theta)$ é a condutividade hidráulica do solo não-saturado, $D(\theta)$ é a difusividade da água no solo e $\theta$ é o conteúdo volumétrico de água no solo.

A equação de Richards unidimensional considerando apenas a infiltração da água na direção vertical já foi largamente explorada na literatura [1], [3], [6]. Entretanto, o escoamento horizontal apresenta particularidades que podem influenciar na solução numérica, conforme apresentado por [5].

$\mathrm{Na}$ tentativa de se obter uma melhor solução aproximada para a direção horizontal, [2] e [4] introduziram uma dependência empírica da difusividade hidráulica em relação a distância $x$ e ao tempo $t$ e usaram a derivada fracionária obtendo a equação de Richards generalizada, dada por

$$
\frac{\partial^{\gamma} \theta}{\partial t^{\gamma}}=\frac{\partial}{\partial x}\left[D_{\gamma}(\theta) \frac{\partial \theta}{\partial x}\right]
$$

onde $\gamma$ é a ordem da derivada fracionária, com $0<\gamma \leq 1$, observando-se resultados numéricos melhores do que aqueles obtidos com a representação clássica da equação de Richards. No entanto, apesar dos bons resultados numéricos obtidos, não há até o momento uma interpretação física para esta dependência.

Neste trabalho apresenta-se um estudo da equação generalizada de Richards (2) para o problema na horizontal, que visa analisar os erros numéricos obtidos quando se reduz o número de passos utilizados no cálculo da derivada fracionária na discretização temporal e mantendo-se o Método de Diferenças Finitas para aproximar as derivadas espaciais. A solução semi-analítica obtida por [5] será comparada com a solução numérica proposta.

Palavras-chave: Equação de Richards, Método de Diferenças Finitas, Derivada fracionária, Modelo generalizado, Infiltração horizontal

*Aluno de Mestrado do Programa de Pós-Graduação em Informática - PPGI-UFRJ 


\section{Referências}

[1] C.A.B. Vasconcellos and J.C.C. Amorim. Numerical Simulation of Unsaturated Flow in Porous Media using a Mass-Conservative Model. XVI Congresso Brasileiro de Engenharia Mecânica COBEM - ABCM, Uberlândia-MG, Brasil, 2001.

[2] I.A. Guerrini and D. Swartzendruber. Fractal characteristics of the horizontal movement of water in soil. Fractals - Complex Geometry Patterns and Scaling in Nature and Society, 2-3 (1994) 465-468.

[3] M.A. Celia, E.T. Bouloutas and R.L. Zarba. A General Mass-Conservative Numerical Solution for the Unsaturated Flow Equation. Water Resources Research, 26-7 (1990) 1483-1496.

[4] Y.A. Pachepsky and D. Timlin. Water Transport in solis as in fractal media. Journal of Hydrology, 204 (1998) 98-107.

[5] Y.A. Pachepskya, D. Timlinb and W. Rawls, Generalized Richards' equation to simulate water transport in unsaturated soils. Journal of Hydrology, 272 (2003) 3-13.

[6] Y. Ma, S. Feng, D. Su, G. Gao and Z. Huo. Modeling water infiltration in a large layered soil column with a modified Green-Ampt model and HYDRUS 1-D. Computers and Eletronics in Agriculture, 1S (2010) S40-S47. 